\title{
SOLVABILITY AND NUMERICAL ALGORITHMS FOR A CLASS OF VARIATIONAL DATA ASSIMILATION PROBLEMS
}

\author{
Guri MarchuK ${ }^{1}$ And Victor ShutyaeV ${ }^{1}$
}

\begin{abstract}
A class of variational data assimilation problems on reconstructing the initial-value functions is considered for the models governed by quasilinear evolution equations. The optimality system is reduced to the equation for the control function. The properties of the control equation are studied and the solvability theorems are proved for linear and quasilinear data assimilation problems. The iterative algorithms for solving the problem are formulated and justified.
\end{abstract}

Mathematics Subject Classification. 65K10.

Received January 14, 2002.

\section{INTRODUCTION}

The investigation of global changes of the Earth System has increased the interest to the observation data assimilation and data processing problems, which are applied to the modeling, retrospective analysis, and forecasting various physical and geophysical processes. From the mathematical standpoint, these problems may be formulated as the optimal control problems. Starting with the studies of Bellman and Pontryagin, these problems attract the attention of many researchers. New essential ideas were contributed to the optimization theory and methods by French mathematical school. In this connection, we must mention the works by J.-L. Lions and his disciples, which became fundamental, dedicated to investigation of problems on controllability, insensitive optimal control, nonlinear sentinels for distributed systems. The general approach (Hilbert Uniqueness Method) developed by J.-L. Lions makes it possible to prove the existence of controls in linear and nonlinear systems.

In this paper, a class of variational data assimilation problems on reconstructing the initial-value functions is considered for the models governed by quasilinear evolution equations. The properties of the equation for the control function are studied and the solvability theorems are proved for linear and quasilinear optimality systems. The iterative algorithms for solving the problem are formulated and justified. The results given in the paper are a logical development of some ideas and aspects concerning the methods for solving the systems considered in $[1,14-16]$.

\section{Statement of the PROBlem}

Let $H$ and $X$ be real separable Hilbert spaces such that $X$ is imbedded into $H$ continuously and densely, $H^{*}$, $X^{*}$ are the spaces adjoint to $H, X$, respectively. We assume that $H \equiv H^{*},(\cdot, \cdot)_{L_{2}(0, T ; H)}=(\cdot, \cdot),\|\cdot\|=(\cdot, \cdot)^{1 / 2}$.

Keywords and phrases: Variational data assimilation, quasilinear evolution problem, optimality system, control equation, solvability, iterative algorithms.

1 Institute of Numerical Mathematics, Russian Academy of Sciences, ul. Gubkina 8, Moscow, 119991 GSP-1 Russia;

e-mail: shutyaev@inm.ras.ru 
Let us consider also the spaces $Y^{0}=L_{2}(0, T ; H), Y=L_{2}(0, T ; X), Y^{*}=L_{2}\left(0, T ; X^{*}\right)$ of abstract functions $f(t)$ with the values in $H, X, X^{*}$, respectively, and the space

$$
\begin{aligned}
& W=\left\{f \in L_{2}(0, T ; X): \frac{\mathrm{d} f}{\mathrm{~d} t} \in L_{2}\left(0, T ; X^{*}\right)\right\}, \\
& \|f\|_{W}=\left(\left\|\frac{\mathrm{d} f}{\mathrm{~d} t}\right\|_{L_{2}\left(0, T ; X^{*}\right)}^{2}+\|f\|_{L_{2}(0, T ; X)}^{2}\right)^{1 / 2} .
\end{aligned}
$$

Let $a(t ; \varphi, \psi)$ be a bilinear form defined for any $t \in[0, T], \varphi, \psi \in X$ and satisfied the inequalities:

$$
\begin{gathered}
|a(t ; \varphi, \psi)| \leq c_{1}\|\varphi\|_{X}\|\psi\|_{X}, \quad c_{1}=\text { const }>0, \\
c_{2}\|\varphi\|_{X}^{2} \leq a(t ; \varphi, \varphi), \quad c_{2}=\text { const }>0, \quad \forall t \in[0, T], \quad \forall \varphi, \psi \in X .
\end{gathered}
$$

By $A(t) \in \mathcal{L}\left(Y, Y^{*}\right)$ we denote the operator generated by this form:

$$
(A(t) \varphi, \psi)_{H}=a(t ; \varphi, \psi) \quad \forall \varphi, \psi \in X .
$$

Consider the following quasilinear evolution problem:

$$
\left\{\begin{array}{l}
\frac{\mathrm{d} \varphi}{\mathrm{d} t}+A(t) \varphi+\tau F(\varphi)=f(t), \quad t \in(0, T) \\
\varphi(0)=u
\end{array}\right.
$$

where $f \in Y^{*}, u \in H, \tau \in\left[-\tau_{0}, \tau_{0}\right]$ is a parameter, $\tau_{0} \in \mathbf{R}^{+}, F(\varphi)$ is a nonlinear Frechet differentiable operator, $F: Y \rightarrow Y^{*}$. Introduce a functional of $u \in H$ of the form:

$$
S(u)=\frac{\alpha}{2}\|u\|_{H}^{2}+\frac{1}{2}\|B \varphi-\widehat{\varphi}\|_{Z}^{2}
$$

where $\alpha=$ const $\geq 0, Z$ is a Hilbert space (observational space) with the scalar product $(\cdot, \cdot)_{Z}$ and the norm $\|\cdot\|_{Z}=(\cdot, \cdot)_{Z}^{1 / 2}, B: Y \rightarrow Z$ is a linear bounded operator, $\widehat{\varphi} \in Z$. The function $\widehat{\varphi}$ is generally determined by $a$ priory observational data. The weight coefficient $\alpha$ is normally called a regularization parameter [23].

Consider the following data assimilation problem: for given $f \in Y^{*}, \widehat{\varphi} \in Z$, find $u \in H, \varphi \in W$ such that

$$
\left\{\begin{aligned}
\frac{\mathrm{d} \varphi}{\mathrm{d} t}+A(t) \varphi+\tau F(\varphi) & =f, \quad t \in(0, T) \\
\varphi(0) & =u \\
S(u) & =\min _{\tilde{u} \in H} S(\tilde{u}) .
\end{aligned}\right.
$$

The problems of the form (1.6) were studied by Pontryagin [18], J.-L. Lions $[8,10]$ and many others (see, e.g. $[1-3,5,7,13-17,19])$.

The necessary optimality condition [8] reduces the problem (1.6) to the system for finding the functions $\varphi, \varphi^{*} \in W, u \in H$, of the form:

$$
\begin{gathered}
\frac{\mathrm{d} \varphi}{\mathrm{d} t}+A(t) \varphi+\tau F(\varphi)=f, t \in(0, T) ; \quad \varphi(0)=u, \\
-\frac{\mathrm{d} \varphi^{*}}{\mathrm{~d} t}+A^{*}(t) \varphi^{*}+\tau\left(F^{\prime}(\varphi)\right)^{*} \varphi^{*}=C \widehat{\varphi}-K \varphi, t \in(0, T) ; \quad \varphi^{*}(T)=0, \\
\alpha u-\varphi^{*}(0)=0,
\end{gathered}
$$


where $\left(F^{\prime}(\varphi)\right)^{*}: Y \rightarrow Y^{*}$ is the operator adjoint to the Frechet derivative of $F$ at the point $\varphi \in W, A^{*}(t)$ : $Y \rightarrow Y^{*}$ is adjoint to $A(t), K: Y \rightarrow Y^{*}, C: Z \rightarrow Y^{*}$ are linear bounded operators, $K=C B, C$ is defined by the equality $(C \theta, \psi)=(\theta, B \psi)_{Z} \forall \theta \in Z, \psi \in Y$, and equations $(1.7,1.8)$ are considered in the space $Y^{*}$.

The solvability of the systems of the form (1.7-1.9) was studied by J.-L. Lions [8,10], and other authors (see, e.g. $[1,11,22]$ etc.) In this paper, following [1,22], we reduce the problem to the equation for the control function, study its properties in linear case, discuss the solvability of the optimality system, and present numerical algorithms to solve it.

\section{Properties of Linear problem}

Consider the problem (1.7-1.9) for $\tau=0$. The solutions of problems $(1.7,1.8)$ for $\tau=0$ may by represented [9] as

$$
\varphi=G_{0} u+G_{1} f, \varphi^{*}=G_{1}^{(T)}(C \widehat{\varphi}-K \varphi)
$$

where $G_{0}: H \rightarrow W, G_{1}: Y^{*} \rightarrow W, G_{1}^{(T)}: Y^{*} \rightarrow W$ are linear bounded operators. Eliminating $\varphi, \varphi^{*}$ from (1.7-1.9) for $\tau=0$, we come to the equation for the control $u$ :

$$
L u=P
$$

where the operator $L: H \rightarrow H$ and the right-hand side $P$ are defined by

$$
L=\alpha E+T_{0} G_{1}^{(T)} K G_{0}, \quad P=T_{0} G_{1}^{(T)} C \widehat{\varphi}-T_{0} G_{1}^{(T)} K G_{1} f
$$

$E$ is the identity operator, $T_{0}: W \rightarrow H$ is the trace operator: $T_{0} \varphi=\left.\varphi\right|_{t=0}$.

Consider the operator $L$ for $\alpha=0$ and denote it by $\bar{L}$. Let $G_{0}: H \rightarrow W$ be the operator from (2.1), where the element $G_{0} u$ is defined as the solution of (1.7) for $\tau=0, f=0$. The following statement holds.

Lemma 2.1. The operator $\bar{L}: H \rightarrow H$ is continuous, self-adjoint, and positive semi-definite:

$$
(\bar{L} v, v)_{H} \geq 0 \quad \forall v \in H .
$$

If the operator $B G_{0}: H \rightarrow Z$ is invertible, the operator $\bar{L}$ is positive: $(\bar{L} v, v)_{H}>0 \quad \forall v \in H, v \neq 0$.

Proof. Let $\rho \in H$ and $\varphi=G_{0} \rho$. Then

$$
\bar{L} \rho=T_{0} G_{1}^{(T)} K \varphi
$$

Since $[9]$

$$
\|\varphi\|_{W} \leq c_{1}\|\rho\|_{H}, \quad c_{1}=\text { const }>0
$$

and similarly for $\varphi^{*}=G_{1}^{(T)} K \varphi$

$$
\left\|\varphi^{*}\right\|_{W} \leq c_{2}\|K \varphi\|_{Y^{*}}, \quad c_{2}=\text { const }>0
$$

and by definition of $K$,

$$
\|K \varphi\|_{Y^{*}} \leq\|C B\|\|\varphi\|_{W}
$$


then

$$
\left\|\varphi^{*}\right\|_{W} \leq c_{3}\|\rho\|_{H}, \quad c_{3}=\text { const }>0 .
$$

The imbedding of $W$ into $C^{0}([0, T] ; H)$ is continuous [9], hence

$$
\left\|T_{0} \varphi^{*}\right\|_{H}=\left\|\varphi^{*}(0)\right\|_{H} \leq c_{4}\left\|\varphi^{*}\right\|_{W}, \quad c_{4}=\mathrm{const}>0,
$$

therefore, for $\bar{L} \rho=T_{0} \varphi^{*}$ we get the inequality

$$
\|\bar{L} \rho\|_{H} \leq\|\rho\|_{H}
$$

which implies the continuity of $\bar{L}$. Obviously, $\bar{L}$ is self-adjoint. The positive definiteness or semi-definiteness of $\bar{L}$ follow from the equalities:

$$
(\bar{L} \rho, \rho)_{H}=\left(T_{0} G_{1}^{(T)} K G_{0} \rho, \rho\right)_{H}=(K \varphi, \varphi)=(B \varphi, B \varphi)_{Z}=\left\|B G_{0} \rho\right\|_{Z}^{2} .
$$

The lemma is proved.

Corollary 2.1. If the operator $B G_{0}: H \rightarrow Z$ is invertible, then

(I) the range $R(\bar{L})$ of the operator $\bar{L}$ is dense in $H$;

(II) the equation $\bar{L} u=P$ is solvable uniquely and densely in $H$.

Remark 2.1. In the case when $Z=\mathbf{R}^{n}, n \in \mathbf{N}$, and the observational operator $B: Y \rightarrow Z$ is given by the formula $B \varphi=\left(\left(\varphi, p_{1}\right), \ldots,\left(\varphi, p_{n}\right)\right)^{T}$, where $p_{i} \in Y^{*}, i=1, \ldots, n$, the operators $C: Z \rightarrow Y, K: Y \rightarrow Y^{*}$ in (1.8) are defined by

$$
C \theta=\sum_{i=1}^{n} \theta_{i} p_{i}, \quad K \varphi=\sum_{i=1}^{n}\left(\varphi, p_{i}\right) p_{i}
$$

where $\theta=\left(\theta_{1}, \ldots, \theta_{n}\right)^{T} \in Z$. Then $(K \varphi, \psi)=(K \psi, \varphi)$ and $(K \psi, \psi)=\sum_{i=1}^{n}\left(\psi, p_{i}\right)^{2} \geq 0 \quad \forall \varphi, \psi \in Y$.

Remark 2.2. In case of "complete observation", when $Z=Y^{0}, B=E$ (the identity operator), we have $C=E, K=E$, and the operator $\bar{L}$ is positive.

Introduce the following additional restriction on the operator $A(t)$ :

Hypothesis (A): For any $p \in Y^{0}$ the solution $\varphi^{*}$ of the adjoint problem

$$
-\frac{\mathrm{d} \varphi^{*}}{\mathrm{~d} t}+A^{*}(t) \varphi^{*}=p, t \in(0, T) ; \quad \varphi^{*}(T)=0
$$

satisfies the inequality $\left\|\varphi^{*}(0)\right\|_{X} \leq c\|p\|_{Y^{0}}, c=$ const $>0$.

Remark 2.3. The Hypothesis (A) is satisfied for a wide class of operators $A(t)$, among them - the second-order elliptic operators in uniformly parabolic problems [6], for instance, in the case when

$$
H=L_{2}(\Omega), \quad X \stackrel{0}{=}{ }_{2}^{1}(\Omega), \quad Y=L_{2}\left(0, T ; \stackrel{0}{W_{2}^{1}}(\Omega)\right), \quad Y^{0}=L_{2}((0, T) \times \Omega)
$$

and $A \in \mathcal{L}\left(Y, Y^{*}\right)$ is the operator defined by the bilinear form:

$$
(A \varphi, \psi)_{H}=a(t ; \varphi, \psi) \quad \forall \varphi, \psi \in \stackrel{0}{W_{2}^{1}}(\Omega)
$$


where $\Omega \subset \mathbf{R}^{n}$ is a bounded domain with a piece-wise regular boundary, $2 \leq n \leq 4$,

$$
\begin{gathered}
a(t ; \varphi, \psi)=\int_{\Omega}\left(\sum_{i, j=1}^{n} a_{i j} \frac{\partial \varphi}{\partial x_{i}} \frac{\partial \psi}{\partial x_{j}}+\sum_{i=1}^{n} a_{i} \frac{\partial \varphi}{\partial x_{i}} \psi+a \varphi \psi\right) \mathrm{d} x, \\
a(t, x), a_{i j}(t, x), a_{i}(t, x) \in L_{\infty}((0, T) \times \Omega), \quad i, j=\overline{1, n}, \quad x \in \Omega, \\
a(t, x) \geq 0, \quad \sum_{i=1}^{n} \frac{\partial a_{i}}{\partial x_{i}}=0, \quad \sum_{i, j=1}^{n} a_{i j} \lambda_{i} \lambda_{j} \geq \gamma \sum_{i=1}^{n} \lambda_{i}^{2} \forall \lambda_{i} \in \mathbf{R}, \quad \gamma=\text { const }>0 .
\end{gathered}
$$

Lemma 2.2. Let $X$ be compactly imbedded into $H$, the Hypothesis $(\mathrm{A})$ be satisfied, and the operator $K: Y^{0}$ $\rightarrow Y^{0}$ be bounded. Then the operator $\bar{L}: H \rightarrow H$ is compact.

Proof. Let us prove that $\bar{L}$ maps a bounded set of $H$ into a compact set. Consider $u \in H$ such that $\|u\|_{H}$ $\leq c_{0}, c_{0}=$ const $>0$. Let $\varphi=G_{0} u, \varphi^{*}=G_{1}^{(T)} K \varphi$, then $\bar{L} u=\varphi^{*}(0)$. Since

$$
\|\varphi\|_{W} \leq c_{1}\|u\|_{H},\left\|\varphi^{*}\right\|_{W} \leq c_{2}\|K \varphi\|_{Y^{*}}, \quad c_{1}, c_{2}=\text { const }>0
$$

and by the Hypothesis (A),

$$
\left\|\varphi^{*}(0)\right\|_{X} \leq c\|K \varphi\|_{Y^{0}}, c=\text { const }>0
$$

then, due to the boundedness of $K: Y^{0} \rightarrow Y^{0}$, we get

$$
\|\bar{L} u\|_{X} \leq c_{3}\|u\|_{H} \leq c_{3} c_{0}
$$

where $c_{3}=$ const $>0$. However, $X$ is compactly imbedded into $H$, hence the set $M=\left\{\bar{L} u:\|u\|_{H} \leq c_{0}\right\}$ is compact in $H$, i.e. the operator $\bar{L}: H \rightarrow H$ is compact.

Corollary 2.2. Under the hypotheses of Lemma 2.2 there exists an orthonormal basis in $H$ of eigenfunctions of the operator $\bar{L}$.

Lemma 2.3. For the spectrum $\sigma(\bar{L})$ of the operator $\bar{L}$ the estimate

$$
0 \leq \sigma(\bar{L}) \leq \nu^{2}\|B\|^{2}
$$

holds with the constant $\nu$ from the inequality $\|\varphi\|_{Y} \leq \nu\|u\|_{H}$, where $u \in H$, and $\varphi=G_{0} u$ is the solution of the problem $\frac{\mathrm{d} \varphi}{\mathrm{d} t}+A(t) \varphi=0, t \in(0, T) ; \quad \varphi(0)=u$.

Proof. To estimate the spectrum of the self-adjoint operator $\bar{L}$ consider $(\bar{L} u, u)$ for $u \in H$. Let $\varphi=G_{0} u$, $\varphi^{*}=G_{1}^{(T)} \varphi$, then

$$
(\bar{L} u, u)_{H}=\left(\varphi^{*}(0), u\right)_{H}=(K \varphi, \varphi)=\|B \varphi\|_{Z}^{2} \leq\|B\|^{2}\|\varphi\|_{Y}^{2} \leq \nu^{2}\|B\|^{2}\|u\|_{H}^{2} .
$$

Hence,

$$
\sigma(\bar{L}) \leq \sup _{u \in H, u \neq 0} \frac{(\bar{L} u, u)}{(u, u)} \leq \nu^{2}\|B\|^{2}
$$

This ends the proof. 
Remark 2.4. In some cases (when, for example, $A$ is self-adjoint and independent of $t$ ), for $\nu$ we can put $\nu=1$. For case of complete observation, from Lemmas 2.1, 2.2 we have the following:

Lemma 2.4. Let $Z=Y^{0}, B=E$ (the identity operator), $X$ be compactly imbedded into $H$ and the Hypothesis (A) be satisfied. Then the following statements hold:

(I) the operator $\bar{L}^{-1}: H \rightarrow H$ exists, being unbounded. Zero is the point of the continuous spectrum of the operator $\bar{L}$;

(II) the equation $\bar{L} u=P$ is solvable in $H$ if and only if

$$
\sum_{k=1}^{\infty} \mu_{k}^{-2}\left(P, u_{k}\right)_{H}^{2}<\infty
$$

where $u_{k}$ is the orthonormal system of the eigenfunctions of the compact operator $\bar{L}$, corresponding to the eigenvalues $\mu_{k}$.

If $K=E$, for the spectrum $\sigma(L)$ of the operator $L$ defined by $(2.2)$ the following estimates hold [21]:

$$
m \leq \sigma(L) \leq M
$$

where

$$
m=\alpha+\int_{0}^{T} \mathrm{e}^{-\int_{0}^{t} \lambda_{\max }(\tau) \mathrm{d} \tau} \mathrm{d} t, \quad M=\alpha+\int_{0}^{T} \mathrm{e}^{-\int_{0}^{t} \lambda_{\min }(\tau) \mathrm{d} \tau} \mathrm{d} t,
$$

and $\lambda_{\min }, \lambda_{\max }$ are the lower and upper bounds, respectively, of the spectrum of the operator $A+A^{*}$.

If $K=E$, and $A(t)=A: H \rightarrow H$ is a linear closed operator independent of time, being unbounded selfadjoint positive definite operator in $H$ with the compact inverse, then the eigenvalues $\mu_{k}$ of the operator $\bar{L}$ are defined by the formula [21]:

$$
\mu_{k}=\frac{1-\mathrm{e}^{-2 \lambda_{k} T}}{2 \lambda_{k}}
$$

where $\lambda_{k}$ are the eigenvalues of the operator $A$. Then in (2.7) $\lambda_{\min }=2 \lambda_{1}, \lambda_{\max }=\infty$, and $m, M$ are given in the explicit form:

$$
m=\alpha, \quad M=\alpha+\frac{1-\mathrm{e}^{-2 \lambda_{1} T}}{2 \lambda_{1}}
$$

where $\lambda_{1}$ is the least eigenvalue of the operator $A$.

\section{Solvability RESUlts}

It follows from Lemma 2.1 that for $\alpha>0$ the operator $L: H \rightarrow H$ is positive definite (i.e. coercive). Then, we come to the solvability theorems for linear and nonlinear problem (1.7-1.9):

Theorem 3.1. Let $f \in Y^{*}, \widehat{\varphi} \in Z$. Then for $\alpha>0$ the problem (1.7-1.9) for $\tau=0$ has a unique solution $\varphi_{0} \in W, \varphi_{0}^{*} \in W, u_{0} \in H$, and the following estimate holds:

$$
\left\|\varphi_{0}\right\|_{W}+\left\|\varphi_{0}^{*}\right\|_{W}+\left\|u_{0}\right\|_{H} \leq c_{0}\left(\|C \widehat{\varphi}\|_{Y^{*}}+\|f\|_{Y^{*}}\right), \quad c_{0}=\text { const }>0 .
$$


Theorem 3.2. Let $u \in H, \widehat{\varphi} \in Z$ and for some $R>0$ the inequalities

$$
\left\|F^{\prime}(\xi)\right\|_{Y \rightarrow Y^{*}} \leq k_{1}, \quad\left\|F^{\prime}(\xi)-F^{\prime}(\eta)\right\|_{Y \rightarrow Y^{*}} \leq k_{2}\|\xi-\eta\|_{W}
$$

are satisfied for any $\xi, \eta \in B\left(\varphi_{0}, R\right)=\left\{\varphi \in Y:\left\|\varphi-\varphi_{0}\right\|_{W} \leq R\right\}$, where $k_{i}=k_{i}\left(\varphi_{0}, R\right)=$ const $>0$. Then for $|\tau| \leq \tau_{0}$, with

$$
\tau_{0}=1 / c_{0}\left[k_{1}+k_{2}\left(R+\left\|\varphi_{0}^{*}\right\|_{W}\right)+\frac{1}{R}\left(\left\|F\left(\varphi_{0}\right)\right\|_{Y^{*}}+k_{1}\left\|\varphi_{0}^{*}\right\|_{W}\right)\right]^{-1}
$$

the problem (1.2-1.4) has a unique solution $\left(\varphi, \varphi^{*}, u\right) \in W \times W \times H$.

Proof. Theorem 3.1 follows from Lemma 2.1 and the well-known results on solvability of linear optimal control problems $[1,8]$. To prove Theorem 3.2, consider the problem for the remainders $\tilde{\varphi}=\varphi-\varphi_{0}, \tilde{\varphi}^{*}=\varphi^{*}-\varphi_{0}^{*}$, $\tilde{u}=u-u_{0}$, where $\left(\varphi_{0}, \varphi_{0}^{*}, u_{0}\right)$ is the solution to the problem $(1.7-1.9)$ for $\tau=0$. The problem for $\tilde{\varphi}, \tilde{\varphi}^{*}, \tilde{u}$ reads:

$$
\begin{gathered}
\frac{\mathrm{d} \tilde{\varphi}}{\mathrm{d} t}+A(t) \tilde{\varphi}+\tau F\left(\varphi_{0}+\tilde{\varphi}\right)=0, t \in(0, T) ; \quad \tilde{\varphi}(0)=\tilde{u}, \\
-\frac{\mathrm{d} \tilde{\varphi}^{*}}{\mathrm{~d} t}+A^{*}(t) \tilde{\varphi}^{*}+\tau\left(F^{\prime}\left(\varphi_{0}+\tilde{\varphi}\right)\right)^{*}\left(\varphi_{0}^{*}+\tilde{\varphi}^{*}\right)=-K \tilde{\varphi}, t \in(0, T) ; \quad \tilde{\varphi}^{*}(T)=0, \\
\alpha \tilde{u}-\tilde{\varphi}^{*}(0)=0 .
\end{gathered}
$$

Consider the following iterative process:

$$
\begin{gathered}
\frac{\mathrm{d} \tilde{\varphi}^{(n+1)}}{\mathrm{d} t}+A(t) \tilde{\varphi}^{(n+1)}+\tau F\left(\tilde{\varphi}^{(n)}+\varphi_{0}\right)=0, t \in(0, T) ; \quad \tilde{\varphi}^{(n+1)}(0)=\tilde{u}^{(n+1)}, \\
-\frac{\mathrm{d} \tilde{\varphi}^{*(n+1)}}{\mathrm{d} t}+A^{*}(t) \tilde{\varphi}^{*(n+1)}+\tau\left(F^{\prime}\left(\tilde{\varphi}^{(n)}+\varphi_{0}\right)\right)^{*}\left(\tilde{\varphi}^{*(n)}+\varphi_{0}^{*}\right)=-K \tilde{\varphi}^{(n+1)}, \tilde{\varphi}^{*(n+1)}(T)=0, \\
\alpha \tilde{u}^{(n+1)}-\tilde{\varphi}^{*(n+1)}(0)=0
\end{gathered}
$$

for $\left\|\tilde{\varphi}^{(0)}\right\|_{W}+\left\|\tilde{\varphi}^{*(0)}\right\|_{W} \leq R$. Since (for a fixed $\left.n\right) \tilde{\varphi}^{(n+1)}, \tilde{\varphi}^{*(n+1)}, \tilde{u}^{(n+1)}$ is the solution of the linear problem, then, in view of $(3.1)$, it is easily seen that

$$
\left\|\tilde{\varphi}^{(n+1)}\right\|_{W}+\left\|\tilde{\varphi}^{*(n+1)}\right\|_{W}+\left\|\tilde{u}^{(n+1)}\right\|_{H} \leq k|\tau|\left(\left\|\tilde{\varphi}^{(n)}\right\|_{W}+\left\|\tilde{\varphi}^{*(n)}\right\|_{W}\right)+f_{0}
$$

where

$$
k=c_{0}\left(k_{1}+k_{2}\left(R+\left\|\varphi_{0}^{*}\right\|_{W}\right)\right), \quad f_{0}=c_{0}|\tau|\left(\left\|F\left(\varphi_{0}\right)\right\|_{Y^{*}}+k_{1}\left\|\varphi_{0}^{*}\right\|_{W}\right) .
$$

By successive use of the last inequality, we get

$$
\begin{aligned}
\left\|\tilde{\varphi}^{(n)}\right\|_{W}+\left\|\tilde{\varphi}^{*(n)}\right\|_{W}+\left\|\tilde{u}^{(n)}\right\|_{H} \leq & (k|\tau|)^{n}\left(\left\|\tilde{\varphi}^{(0)}\right\|_{W}+\left\|\tilde{\varphi}^{*(0)}\right\|_{W}\right) \\
& +\frac{1-(k|\tau|)^{n}}{1-k|\tau|} f_{0} \leq(k|\tau|)^{n} R+\frac{1-(k|\tau|)^{n}}{1-k|\tau|} f_{0} \leq R
\end{aligned}
$$


if $|\tau| \leq \tau_{0}$. Then, consider the problem for $\tilde{\varphi}^{(n+1)}-\tilde{\varphi}^{(n)}, \tilde{\varphi}^{*(n+1)}-\tilde{\varphi}^{*(n)}, \tilde{u}^{(n+1)}-\tilde{u}^{(n)}$. This leads to the estimate:

$\left\|\tilde{\varphi}^{(n+1)}-\tilde{\varphi}^{(n)}\right\|_{W}+\left\|\tilde{\varphi}^{*(n+1)}-\tilde{\varphi}^{*(n)}\right\|_{W}+\left\|\tilde{u}^{(n+1)}-\tilde{u}^{(n)}\right\|_{H} \leq k|\tau|\left(\left\|\tilde{\varphi}^{(n)}-\tilde{\varphi}^{(n-1)}\right\|_{W}+\left\|\tilde{\varphi}^{*(n)}-\tilde{\varphi}^{*(n-1)}\right\|_{W}\right)$

which implies

$$
\tilde{\varphi}^{(n)} \rightarrow \tilde{\varphi}, \quad \tilde{\varphi}^{*(n)} \rightarrow \tilde{\varphi}^{*}, \quad \tilde{u}^{(n)} \rightarrow \tilde{u} \text { as } n \rightarrow \infty, \text { for }|\tau| \leq \tau_{0}
$$

where $\tilde{\varphi}, \tilde{\varphi}^{*}, \tilde{u}$ is the solution to the problem $(3.4-3.6)$, and the convergence rate estimate holds:

$$
\left\|\tilde{\varphi}^{(n)}-\tilde{\varphi}\right\|_{W}+\left\|\tilde{\varphi}^{*(n)}-\tilde{\varphi}^{*}\right\|_{W}+\left\|\tilde{u}^{(n)}-\tilde{u}\right\|_{H} \leq c \frac{(k|\tau|)^{n}}{1-k|\tau|}
$$

with $c=$ const $>0$. It is easily seen that for $|\tau| \leq \tau_{0}$ this solution is unique and satisfies the condition $\|\tilde{\varphi}\|_{W}+\left\|\tilde{\varphi}^{*}\right\|_{W}+\|\tilde{u}\|_{H} \leq R$. Thus, under the hypotheses of theorem, there exists a unique solution of the problem (1.7-1.9). Theorem is proved.

Remark 3.1. If the operator $F(\varphi)$ is analytic, then the functions $\left(\varphi, \varphi^{*}, u\right)$ are represented as the series in the powers of $\tau$ :

$$
\varphi=\varphi_{0}+\sum_{i=1}^{\infty} \tau^{i} \varphi_{i}, \quad \varphi^{*}=\varphi_{0}^{*}+\sum_{i=1}^{\infty} \tau^{i} \varphi_{i}^{*}, \quad u=u_{0}+\sum_{i=1}^{\infty} \tau^{i} u_{i}
$$

convergent for $|\tau|<\tau_{0}$ in $W, W, H$, respectively, where $\varphi_{i}, \varphi_{i}^{*}, u_{i}$ may be found by the small parameter method [11].

The interval of the values of $\tau$, for which the nonlinear problem (1.2-1.4) is solvable, may be enlarged by introducing additional restrictions on the functions $\widehat{\varphi}, f$, following [22].

\section{Numerical Algorithms}

To solve (1.7-1.9) one may use the successive approximation method (3.7-3.9). Each step of this method involves a linear data assimilation problem of the form $(1.7-1.9)$ for $\tau=0$. To solve it we consider a class of iterative algorithms:

$$
\begin{gathered}
\frac{\mathrm{d} \varphi^{k}}{\mathrm{~d} t}+A(t) \varphi^{k}=f, t \in(0, T) ; \quad \varphi^{k}(0)=u^{k}, \\
-\frac{\mathrm{d} \varphi^{* k}}{\mathrm{~d} t}+A^{*}(t) \varphi^{* k}=C \widehat{\varphi}-K \varphi^{k}, t \in(0, T) ; \quad \varphi^{* k}(T)=0, \\
u^{k+1}=u^{k}-\alpha_{k+1} B_{k}\left(\alpha u^{k}-\left.\varphi^{* k}\right|_{t=0}\right)+\beta_{k+1} C_{k}\left(u^{k}-u^{k-1}\right),
\end{gathered}
$$

where $B_{k}, C_{k}: H \rightarrow H$ are some operators, and $\alpha_{k+1}, \beta_{k+1}$ the iterative parameters.

Let $\gamma=\nu^{2}\|B\|^{2}$ with $\nu$ defined in (2.5). We introduce the following notations:

$$
\begin{gathered}
\tau_{o p t}=2(2 \alpha+\gamma)^{-1}, \quad \theta=(2 \alpha+\gamma) \gamma^{-1}, \\
\tau_{k}=2\left(2 \alpha+\gamma-\gamma \cos \omega_{k} \pi\right)^{-1}, \quad k=1,2, \ldots, s,
\end{gathered}
$$




$$
\begin{gathered}
\alpha_{k+1}=\left\{\begin{array}{c}
2(2 \alpha+\gamma)^{-1}, k=0 \\
4 \gamma^{-1} \frac{T_{k}(\theta)}{T_{k+1}(\theta)}, k>0
\end{array}\right. \\
\beta_{k+1}=\left\{\begin{array}{c}
0, k=0 \\
\frac{T_{k-1}(\theta)}{T_{k+1}(\theta)}, k>0,
\end{array}\right. \\
e_{k}=\left\{\begin{array}{c}
0, \quad k=0 \\
p_{k}\left\|\xi^{k}\right\|_{H}^{2} /\left\|\xi^{k-1}\right\|_{H}^{2}, k>0,
\end{array}\right. \\
p_{k+1}=\alpha+\left(K \eta^{k}, \eta^{k}\right) /\left\|\xi^{k}\right\|_{H}^{2}-e_{k}, \quad k=0,1, \ldots,
\end{gathered}
$$

where $\omega_{k}=(2 i-1) / 2 s, T_{k}$ is the $k$-th degree Chebyshev polynomial of the first kind, $\xi^{k}=\alpha u^{k}-\varphi^{* k}(0)$, and $\eta^{k}$ is the solution of the problem $\frac{\mathrm{d} \eta^{k}}{\mathrm{~d} t}+A \eta^{k}=0, t \in(0, T) ; \quad \eta^{k}(0)=\xi^{k}$.

Theorem 4.1. (I) If $\alpha_{k+1}=\tau, B_{k}=E, \beta_{k+1}=0,0<\tau<2 /(\alpha+\gamma)$, then the iterative process (4.1-4.3) is convergent. For $\tau=\tau_{\text {opt }}$ defined by (4.4) the following convergence rate estimates are valid:

$$
\left\|\varphi-\varphi^{k}\right\|_{W} \leq c_{1} q_{k}, \quad\left\|\varphi^{*}-\varphi^{* k}\right\|_{W} \leq c_{2} q_{k}, \quad\left\|u-u^{k}\right\|_{H} \leq c_{3} q_{k}
$$

where $q_{k}=1 / \theta^{k}, \theta$ is given by (4.4), and the constants $c_{1}, c_{2}, c_{3}, c_{4}$ do not depend on the number of iterations and on the functions $\varphi, \varphi^{k}, \varphi^{*}, \varphi^{* k}, u, u^{k}, k>0$.

(II) If $B_{k}=E, \beta_{k+1}=0$, and $\alpha_{k+1}=\tau_{k}$, where the parameters $\tau_{k}$ are defined by (4.5) and repeated cyclically with the period $s$, then the error in the iterative process (4.1-4.3) is suppressed after each cycle of the length s. After $k=l s$ iterations the error estimates (4.9) are valid with $q_{k}=\left(T_{s}(\theta)\right)^{-l}$.

(III) If $B_{k}=C_{k}=E$ and $\alpha_{k+1}, \beta_{k+1}$ are defined by (4.6), then the error in the algorithm (4.1-4.3) is suppressed for each $k \geq 1$, and the estimates (4.9) hold for $q_{k}=\left(T_{k}(\theta)\right)^{-1}$.

(IV) If $B_{k}=C_{k}=E$ and $\alpha_{k+1}=1 / p_{k+1}, \beta_{k+1}=e_{k} / p_{k+1}$, where $e_{k}, p_{k+1}$ are defined by $(4.7,4.8)$, then the iterative process (4.1-4.3) is convergent, and the convergence rate estimates (4.9) are valid with $q_{k}=\left(T_{k}(\theta)\right)^{-1}$.

Proof. It is not difficult to show [14] that the iterative process (4.1-4.3) is equivalent to the following iterative algorithm

$$
u^{k+1}=u^{k}-\alpha_{k+1} B_{k}\left(L u^{k}-P\right)+\beta_{k+1} C_{k}\left(u^{k}-u^{k-1}\right)
$$

for solving the control equation $L u=P$, where $L$ and $P$ are defined in (2.2).

According to Lemma 2.3, the bounds of the spectrum of the control operator $L$ are given by

$$
m \stackrel{\text { def }}{=} \inf _{u \in H, u \neq 0} \frac{(L u, u)}{(u, u)} \geq \alpha, \quad M \stackrel{\text { def }}{=} \sup _{u \in H, u \neq 0} \frac{(L u, u)}{(u, u)} \leq \alpha+\nu^{2}\|B\|^{2} .
$$


Thus, for $\alpha>0$ for solving the equation $L u=P$ we may use the well-known iterative algorithms with optimal choice of parameters. The theory of these methods is well developed [12]. Taking into account the explicit form of the bounds for $m$ and $M$ from (4.11) and applying for the equation $L u=P$ the simple iterative method, the Chebyshev acceleration methods (s-cyclic and two-step ones), and the conjugate gradient method in the form (4.10), we arrive at the conclusions of theorem, using the well-known convergence results [12] for these methods.

Remark 4.1. In case of complete observation, when $Z=Y^{0}, B=E, K=E$, for the spectrum of the operator $L$ the estimates (2.7) are valid, and in the formulas for iterative parameters (4.4-4.8) we may take

$$
\gamma=\int_{0}^{T} \mathrm{e}^{-\int_{0}^{t} \lambda_{\min }(\tau) \mathrm{d} \tau} \mathrm{d} t .
$$

If, moreover, the operator $A$ is self-adjoint and independent of $t$, we can put, due to $(2.8), \gamma=\left(1-\mathrm{e}^{-2 \lambda_{1} T}\right) /\left(2 \lambda_{1}\right)$.

The numerical analysis of the above-formulated iterative algorithms has been done in [17] for the data assimilation problem with a linear parabolic state equation.

In case $\alpha_{k}=1 / \alpha, B_{k}=E, \beta_{k}=0$, the iterative algorithm (4.1-4.3) coincides with the Krylov-Chernousko method [4].

\section{REFERENCES}

[1] V.I. Agoshkov and G.I. Marchuk, On solvability and numerical solution of data assimilation problems. Russ. J. Numer. Analys. Math. Modelling 8 (1993) 1-16.

[2] R. Bellman, Dynamic Programming. Princeton Univ. Press, New Jersey (1957).

[3] R. Glowinski and J.-L. Lions, Exact and approximate controllability for distributed parameter systems. Acta Numerica 1 (1994) 269-378.

[4] I.A. Krylov and F.L. Chernousko, On a successive approximation method for solving optimal control problems. Zh. Vychisl. Mat. Mat. Fiz. 2 (1962) 1132-1139 (in Russian).

[5] A.B. Kurzhanskii and A.Yu. Khapalov, An observation theory for distributed-parameter systems. J. Math. Syst. Estimat. Control 1 (1991) 389-440.

[6] O.A. Ladyzhenskaya, V.A. Solonnikov and N.N. Uraltseva, Linear and Quasilinear Equations of Parabolic Type. Nauka, Moscow (1967) (in Russian).

[7] F.X. Le Dimet and O. Talagrand, Variational algorithms for analysis and assimilation of meteorological observations: Theoretical aspects. Tellus 38A (1986) 97-110.

[8] J.-L. Lions, Contrôle Optimal des Systèmes Gouvernés par des Équations aux Dérivées Partielles. Dunod, Paris (1968).

[9] J.-L. Lions and E. Magenes, Problémes aux Limites non Homogènes et Applications. Dunod, Paris (1968).

[10] J.-L. Lions, On controllability of distributed system. Proc. Natl. Acad. Sci. USA 94 (1997) 4828-4835.

[11] G.I. Marchuk, V.I. Agoshkov and V.P. Shutyaev, Adjoint Equations and Perturbation Algorithms in Nonlinear Problems. CRC Press Inc., New York (1996).

[12] G.I. Marchuk and V.I. Lebedev, Numerical Methods in the Theory of Neutron Transport. Harwood Academic Publishers, New York (1986).

[13] G.I. Marchuk and V.V. Penenko, Application of optimization methods to the problem of mathematical simulation of atmospheric processes and environment, in Modelling and Optimization of Complex Systems, Proc. of the IFIP-TC7 Work. Conf. Springer, New York (1978) 240-252.

[14] G.I. Marchuk and V.P. Shutyaev, Iteration methods for solving a data assimilation problem. Russ. J. Numer. Anal. Math. Modelling 9 (1994) 265-279.

[15] G. Marchuk, V. Shutyaev and V. Zalesny, Approaches to the solution of data assimilation problems, in Optimal Control and Partial Differential Equations. IOS Press, Amsterdam (2001) 489-497.

[16] G.I. Marchuk and V.B. Zalesny, A numerical technique for geophysical data assimilation problem using Pontryagin's principle and splitting-up method. Russ. J. Numer. Anal. Math. Modelling 8 (1993) 311-326.

[17] E.I. Parmuzin and V.P. Shutyaev, Numerical analysis of iterative methods for solving evolution data assimilation problems. Russ. J. Numer. Anal. Math. Modelling 14 (1999) 265-274. 
[18] L.S. Pontryagin, V.G. Boltyanskii, R.V. Gamkrelidze and E.F. Mischenko, The Mathematical Theory of Optimal Processes. John Wiley, New York (1962).

[19] Y.K. Sasaki, Some basic formalisms in numerical variational analysis. Mon. Wea. Rev. 98 (1970) 857-883.

[20] V.P. Shutyaev, On a class of insensitive control problems. Control and Cybernetics 23 (1994) 257-266.

[21] V.P. Shutyaev, Some properties of the control operator in a data assimilation problem and algorithms for its solution. Differential Equations 31 (1995) 2035-2041.

[22] V.P. Shutyaev, On data assimilation in a scale of Hilbert spaces. Differential Equations 34 (1998) 383-389.

[23] A.N. Tikhonov, On the solution of ill-posed problems and the regularization method. Dokl. Akad. Nauk SSSR 151 (1963) 501-504. 